\title{
Modeling of a Chaotic Gyrostat System and Mechanism Analysis of Dynamics Using Force and Energy
}

\author{
Guoyuan Qi iD and Xiaogang Yang \\ Tianjin Key Laboratory of Advanced Technology of Electrical Engineering and Energy, School of Electrical Engineering and Automation, \\ Tianjin Polytechnic University, Tianjin 300387, China
}

Correspondence should be addressed to Guoyuan Qi; guoyuanqisa@qq.com

Received 9 April 2019; Accepted 13 June 2019; Published 2 July 2019

Academic Editor: Yu-Wang Chen

Copyright (C) 2019 Guoyuan Qi and Xiaogang Yang. This is an open access article distributed under the Creative Commons Attribution License, which permits unrestricted use, distribution, and reproduction in any medium, provided the original work is properly cited.

\begin{abstract}
Incorporating both viscous friction torque and external torque, a mathematical model of a gyrostat system consisting of three rotors and a fixed outer frame is configured. This model is transformed into a Kolmogorov-type system for force analysis. The force field in the gyrostat system includes four different torques-inertial, internal, dissipative, and external. Correspondingly, four different energies are identified and the interconversion of energies is analyzed. The Casimir power being equivalent to the error power between the supplied power and the dissipative power is found and used to analyze the mechanism underlying the different dynamical behaviors. A four-wing chaotic attractor is found when the gyrostat is subject to a combination of inertial, internal, and dissipative torques as well as the addition of an external torque. The bifurcation of the Casimir power and leaps in Casimir energy level are used as indicators of the different definitive dynamics, which is demonstrated to be identical to that appearing in the state bifurcation and the Lyapunov exponent spectrum.
\end{abstract}

\section{Introduction}

The dynamics of the gyrostat model have been extensively studied over the past two centuries, and they are important for applications in the gyrostat dynamics of satellites, spacecraft, aircraft, and robots. The earlier achievements concerning the equations of motion for a gyrostat and the analytical solutions of the equations governing the gyrostat dynamics were proposed by Wittenberg [1]. Holmes and Marsden [2,3] studied Smale's horseshoe maps of Hamiltonian systems in two degrees of freedom with positive and negative damping and established the existence of such maps. From the research results of Holmes and Marsden [3], Krishnaprasad [4] established the Lie-Poisson structures for a dual-spin spacecraft with driven and free-spinning rotors and analyzed the asymptotic stability in the presence of damping. Liu, Tong, and coworkers [5-7] studied the global motion of an asymmetrical gyrostat with internal and external energy dissipations and the global motion of rigid bodies with small dissipative disturbance torques. By applying Melnikov's method, the intersection of the stable and unstable manifolds of the system's hyperbolic point was determined to highlight the ranges of the physical parameters corresponding to the occurrences of chaos [6]. In studying the chaotic characteristics of the gyrostat system using Melnikov's method [8-14], the gyrostat system was analyzed by deriving its Melnikov equation and obtaining the transversal intersections of the stable and unstable manifolds. Usually, the Melnikov equation provides the necessary conditions for the existence of chaotic motion $[15,16]$.

However, these features of the model cannot identify the mechanism or explain the cause of the dynamic behavior generated and the energy exchange corresponding to the different force fields in the gyrostat system. The gyrostat motion is forced by comprehensive forces, and these forces are coupled. To find the mechanism underlying its definitive behavior, force and energy analysis is necessary. Arnold [17] presented a Kolmogorov model describing a dissipativeforced dynamic system or hydrodynamic instability with a Hamiltonian function. The importance is that the model decomposes the total forces (torques) into four classes: inertial, internal, dissipative, and external. A simple force 
(torque) corresponds to a simple behavior for the given system; a comprehensive force (torque) elicits complex behavior. If a system is transformed into the Kolmogorov model, its underlying mechanism is able to be uncovered by establishing the link between force (torque) and definitive behavior. Pasini and Pelino [18] transformed the Lorenz system into a Kolmogorov model, thereby providing the force analysis of the Lorenz system. Casimir energy plays a vital role in the energy analysis of rigid body systems. By the Casimir energy method of geometric mechanics, Pelino and coworkers [19] presented the energy cycle of the Lorenz system employing the Kolmogorov model. Qi and Liang $[20,21]$ studied the mechanics of the Qi fourwing chaotic system and the Qi chaotic system to perform a forcing analysis, where the variables of the systems are interpreted as angular momenta of a forced-dissipative rigid body. Furthermore, Qi and Zhang [22, 23] gave an analysis of energy cycling and bounds for the Qi and the Qi fourwing chaotic systems, the correspondence between torque and energy was found, and the links between the energetics and chaotic properties and the system definitive behaviors have been found. Qi and coworkers [24, 25] used the Kolmogorov model to perform a force and energy analysis on actual physical models such as the brushless DC motor and permanent magnet synchronous motor. In the dynamic analysis of various physical models [26-30], the bifurcation dynamics play an important role in the study of the motion mechanism of the dynamics. In the study of the plasma system model [31], Yang and Qi used the bifurcation of the Casimir power to evaluate the evolution of the system's orbit.

The artificial satellite is one of the actual mechanical models of the gyrostats. The dynamics of different structural satellites, including the dual-spin satellite and the three-axis stabilized satellite, have received great attention in many studies. Based on the research of three-axis stabilized satellites $[10,32]$, we present a gyrostat system with a three-axis rotor. Thus, a mathematical model of the gyrostat system with the three-axis stabilized satellites as background is developed and is transformed into a Kolmogorov model. The object studied is a gyrostat system under external excitations and disturbances with the internal structural terms of the system being highly coupled. The error torque and power between the dissipative and external torques are found and used to reveal the cause of the different definitive behaviors of the system. A correlation is established between the error power and Casimir power.

The remainder of the paper is organized as follows. Sections 2-4 contain a description of the dynamic equations of the gyrostat and models for the external and disturbance torques, the Kolmogorov transformation of the gyrostat system model, and the four energy forms and their cycles. Section 5 analyzes the energy cycle between the different dynamic modes and reveals the causes of chaos and other definitive behaviors. In Section 6, the dynamic characteristics of the orbit of the system are analyzed using the bifurcation diagram, the Lyapunov exponent, and the bifurcation diagram of the Casimir power. Finally, some conclusions are presented in Section 7.

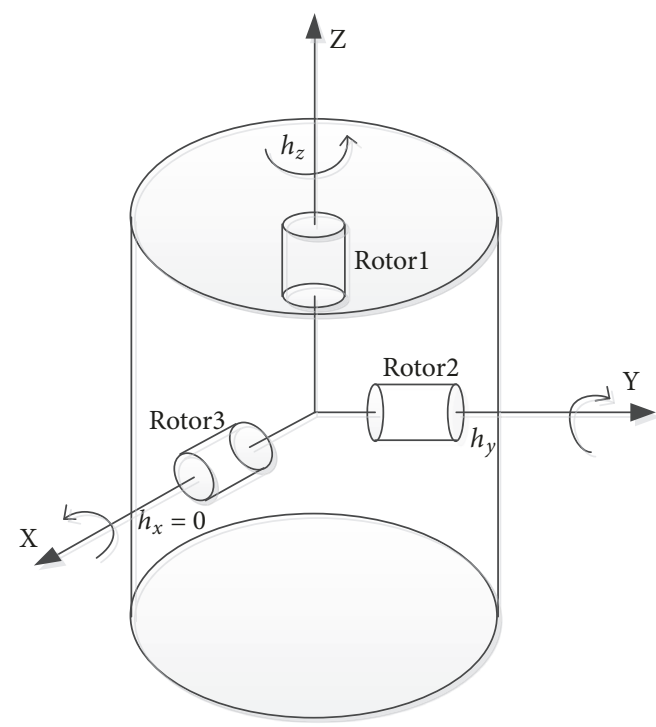

FIGURE 1: Gyrostat system model.

\section{Description of the System Equations}

The general dynamic equations of the gyrostat system required in subsequent sections are provided. Moreover, the relations concerning the disturbance torques are briefly reviewed.

2.1. Physical Model and Dynamic Equations. A rigid body consisting of rotors fixed to a main platform is often referred to as a gyrostat. We shall further specialize this configuration slightly and assume that three of the rotors are inertial and axisymmetric, each spinning about its axis with respect to the main body (see Figure 1). Euler's dynamical equations of rotational motion for a disturbed gyrostat are [1]

$$
\begin{aligned}
& I_{x} \frac{d \omega_{x}}{d t}=\left(I_{\mathrm{y}}-I_{z}\right) \omega_{y} \omega_{z}-\omega_{y} h_{z}+\omega_{z} h_{y}-T_{x}+L_{x}, \\
& I_{y} \frac{d \omega_{y}}{d t}=\left(I_{z}-I_{x}\right) \omega_{z} \omega_{x}-\omega_{z} h_{x}+\omega_{x} h_{z}-T_{y}+L_{x}, \\
& I_{z} \frac{d \omega_{z}}{d t}=\left(I_{x}-I_{y}\right) \omega_{x} \omega_{y}-\omega_{x} h_{y}+\omega_{y} h_{x}-T_{z}+L_{x},
\end{aligned}
$$

where $\boldsymbol{\omega}=\left[\begin{array}{lll}\omega_{x} & \omega_{y} & \omega_{z}\end{array}\right]^{\mathrm{T}}$ is the angular velocity vector; $I_{x}, I_{y}$, and $I_{z}$ are the principal moments of inertia of the gyrostat (including rotors) in the body axis frame-they are constants depending on the shape and mass distribution of the body; $h_{x}, h_{y}$, and $h_{z}$ are the constant components of the total angular momentum vector of the reaction rotors with respect to the body-fixed coordinate system; $T_{x}, T_{y}$, and $T_{z}$ are components of the vector of disturbance torques; and $L_{x}$, $L_{y}$, and $L_{z}$ are external torques.

2.2. Disturbance Torques. The expressions specifying disturbance torques that exist in the gyrostat are ([9])

$$
T_{x}=\varepsilon\left(\mu_{x x} \omega_{x}+\mu_{x y} \omega_{y}+\mu_{x z} \omega_{z}\right),
$$




$$
\begin{aligned}
& T_{y}=\varepsilon\left(\mu_{y x} \omega_{x}+\mu_{y y} \omega_{y}+\mu_{y z} \omega_{z}\right), \\
& T_{z}=\varepsilon\left(\mu_{z x} \omega_{x}+\mu_{z y} \omega_{y}+\mu_{z z} \omega_{z}\right),
\end{aligned}
$$

where $\mu_{i j}(i, j=x, y, z)$ are the damping constants and $\varepsilon$ is a numerical parameter. Assuming that any rotor damping relates only to its own axis (not to any other one) and remains constant, then, $\mu_{x y}=\mu_{x z}=\mu_{y x}=\mu_{y z}=\mu_{z y}=\mu_{z x}=0$; moreover, we set $\mu_{x x}=\mu_{x}, \mu_{y y}=-\mu_{y}, \mu_{z z}=\mu_{z}$, and $\varepsilon=1$. $\mu_{i j}(i, j=x, y, z)$ is the damping coefficient with $\mu_{i j}>0$ meaning that the energy is being dissipated from the system and $\mu_{i j}<0$ meaning that the energy is supplied to the system [33]. Here, $\mu_{y y}=-\mu_{y}$ means that the damping is negative, which is equivalent to a supplied force of the angular velocity $\omega_{y}$ along the $y$-axis direction. In practice, to implement the negative damping, we can install a motor for rotor 2 , and it rotates the axis $y$ with supplied torque $-\mu_{y} \omega_{y}$.

The disturbance torque is expressed as follows:

$$
\left[\begin{array}{c}
T_{x} \\
T_{y} \\
T_{z}
\end{array}\right]=\left[\begin{array}{ccc}
\mu_{x} & 0 & 0 \\
0 & -\mu_{y} & 0 \\
0 & 0 & \mu_{z}
\end{array}\right]\left[\begin{array}{l}
\omega_{x} \\
\omega_{y} \\
\omega_{z}
\end{array}\right] .
$$

The focus here is to study the attitude motion of the gyrostat system for the particular case $h_{x}=0, h_{y} \neq 0, h_{z} \neq 0, I_{x} \neq$ $I_{y} \neq I_{y}$ as studied in [12]. Straightforwardly, as given in [12], we obtain from (1)-(3)

$$
\begin{aligned}
& I_{x} \frac{d \omega_{x}}{d t}=\left(I_{y}-I_{z}\right) \omega_{y} \omega_{z}-\omega_{y} h_{z}+\omega_{z} h_{y}-\mu_{x} \omega_{x}+L_{x}, \\
& I_{y} \frac{d \omega_{y}}{d t}=\left(I_{z}-I_{x}\right) \omega_{z} \omega_{x}+\omega_{x} h_{z}+\mu_{y} \omega_{y}+L_{y} \\
& I_{z} \frac{d \omega_{z}}{d t}=\left(I_{x}-I_{y}\right) \omega_{x} \omega_{y}-\omega_{x} h_{y}-\mu_{z} \omega_{z}+L_{z} .
\end{aligned}
$$

\section{Force Field of the Gyrostat}

Each variable or parameter in the gyrostat system has its own physical meaning. To study the mechanical properties of the gyrostat system, we introduce the Kolmogorov model and the Euler equation. Arnold [17] presented a Kolmogorov model describing dissipative-forced dynamical systems given in the form

$$
\dot{\mathbf{x}}=\{\mathbf{x}, H\}-\boldsymbol{\Lambda} \mathbf{x}+\mathbf{f},
$$

where $\mathbf{x}=\left[\begin{array}{lll}x_{1} & x_{2} & x_{3}\end{array}\right]^{\mathrm{T}},\{\cdot, \cdot\}$ represents the Lie-Poisson structure of the kinetic energy part of the Hamiltonian function $H$ of a system, $\Lambda$ is a positive definite diagonal matrix, $-\Lambda \mathbf{x}$ represents the dissipation (or disturbance torques), and f represents the external torque. For a three-dimensional system, the Lie-Poisson bracket on functions of $\mathbf{x}$ takes the form [34]

$$
\{F, G\}(\mathbf{x})=-\langle\mathbf{x}, \nabla F \times \nabla G\rangle,
$$

where $F, G \in C^{\infty}(g *), g$ is a Lie algebra and $\langle$,$\rangle is the inner$ product. Hence, from (6) we have

$$
\{\mathbf{x}, H\}=-\mathbf{x} \cdot(\nabla \mathbf{x} \times \nabla H)=\mathbf{x} \times \nabla H .
$$

In the absence of external forces, the Euler equations for the rotational dynamics of a rigid body about its center of mass are [34]

$$
\begin{aligned}
& \dot{L}_{1}=\left(I_{2}-I_{3}\right) \omega_{2} \omega_{3}=\left(\Pi_{3}-\Pi_{2}\right) L_{2} L_{3}, \\
& \dot{L}_{2}=\left(I_{3}-I_{1}\right) \omega_{1} \omega_{3}=\left(\Pi_{1}-\Pi_{3}\right) L_{1} L_{3}, \\
& \dot{L}_{3}=\left(I_{1}-I_{2}\right) \omega_{1} \omega_{2}=\left(\Pi_{2}-\Pi_{1}\right) L_{1} L_{2},
\end{aligned}
$$

where $\Pi_{i}=I_{i}^{-1}(i=1,2,3), I_{i}$ is the principle moment of inertia for the group $\mathrm{SO}(3)$, and $L_{i}$ is the angular momentum satisfying $L_{i}=I_{i} \omega_{i}$, with $\omega_{i}$ being the angular velocity corresponding $I_{i}$.

To satisfy the skew-symmetric property of $\{\mathbf{x}, H\}$ for system (4), we set $\overline{\boldsymbol{\omega}}=\left[\begin{array}{lll}\bar{\omega}_{x} & \bar{\omega}_{y} & \bar{\omega}_{z}\end{array}\right]^{\mathrm{T}}, \boldsymbol{\omega}=\left[\begin{array}{lll}\omega_{x} & \omega_{y} & \omega_{z}\end{array}\right]^{\mathrm{T}}$, and $M=\operatorname{diag}\left(\begin{array}{llll}I_{\mathrm{x}} & I_{y} & I_{z}\end{array}\right)$. We introduce the reparameterization

$$
\bar{\omega}=\sqrt{M} \omega
$$

into the original gyrostat system (4) to obtain

$$
\begin{aligned}
\frac{d \bar{\omega}_{x}}{d t}= & \frac{\left(I_{y}-I_{z}\right)}{\sqrt{I_{x} I_{y} I_{z}}} \bar{\omega}_{y} \bar{\omega}_{z}-\frac{h_{z}}{\sqrt{I_{x} I_{y}}} \bar{\omega}_{y}+\frac{h_{y}}{\sqrt{I_{x} I_{z}}} \bar{\omega}_{z} \\
& -\frac{\mu_{x}}{I_{x}} \bar{\omega}_{x}+\frac{L_{x}}{\sqrt{I_{x}}}, \\
\frac{d \bar{\omega}_{y}}{d t}= & \frac{\left(I_{z}-I_{x}\right)}{\sqrt{I_{x} I_{y} I_{z}}} \bar{\omega}_{x} \bar{\omega}_{z}+\frac{h_{z}}{\sqrt{I_{x} I_{y}}} \bar{\omega}_{x}+\frac{\mu_{y}}{I_{y}} \bar{\omega}_{y}+\frac{L_{y}}{\sqrt{I_{y}}}, \\
\frac{d \bar{\omega}_{z}}{d t}= & \frac{\left(I_{x}-I_{y}\right)}{\sqrt{I_{x} I_{y} I_{z}}} \bar{\omega}_{x} \bar{\omega}_{y}-\frac{h_{y}}{\sqrt{I_{x} I_{z}}} \bar{\omega}_{x}-\frac{\mu_{z}}{I_{z}} \bar{\omega}_{z}+\frac{L_{z}}{\sqrt{I_{z}}} .
\end{aligned}
$$

Setting

$$
\begin{aligned}
& \Pi_{3}-\Pi_{2}=\frac{\left(I_{y}-I_{z}\right)}{\sqrt{I_{x} I_{z} I_{y}}}, \\
& \Pi_{1}-\Pi_{3}=\frac{\left(I_{z}-I_{x}\right)}{\sqrt{I_{x} I_{z} I_{y}}}, \\
& \Pi_{2}-\Pi_{1}=\frac{\left(I_{x}-I_{y}\right)}{\sqrt{I_{x} I_{z} I_{y}}},
\end{aligned}
$$

we then find

$$
\begin{aligned}
& {\left[\begin{array}{lll}
\Pi_{1} & \Pi_{2} & \Pi_{3}
\end{array}\right]^{\mathrm{T}}} \\
& \quad=\left[\begin{array}{ll}
1 & \left.1+\frac{\left(I_{x}-I_{y}\right)}{\sqrt{I_{x} I_{z} I_{y}}} 1+\frac{\left(I_{x}-I_{z}\right)}{\sqrt{I_{x} I_{z} I_{y}}}\right]^{\mathrm{T}} .
\end{array}\right.
\end{aligned}
$$


Using (9), the kinetic energy is

$$
\begin{aligned}
K & =\frac{1}{2}\left(\Pi_{1} \bar{\omega}_{x}^{2}+\Pi_{2} \bar{\omega}_{y}^{2}+\Pi_{3} \bar{\omega}_{z}^{2}\right) \\
& =\frac{1}{2}\left(\Pi_{1} I_{x} \omega_{x}^{2}+\Pi_{2} I_{y} \omega_{y}^{2}+\Pi_{3} I_{z} \omega_{z}^{2}\right) .
\end{aligned}
$$

Setting the internal forcing as

$$
\boldsymbol{\beta}=\left[0-\frac{h_{y}}{\sqrt{I_{x} I_{z}}}-\frac{h_{z}}{\sqrt{I_{x} I_{y}}}\right]^{\mathrm{T}},
$$

then the potential energy is

$$
\begin{aligned}
U & =\langle\overline{\boldsymbol{\omega}}, \boldsymbol{\beta}\rangle=-\frac{h_{z}}{\sqrt{I_{x} I_{y}}} \bar{\omega}_{z}-\frac{h_{y}}{\sqrt{I_{x} I_{z}}} \bar{\omega}_{y} \\
& =-\frac{h_{z} \sqrt{I_{z}}}{\sqrt{I_{x} I_{y}}} \omega_{z}-\frac{h_{y} \sqrt{I_{y}}}{\sqrt{I_{x} I_{z}}} \omega_{y} .
\end{aligned}
$$

The Hamiltonian function is

$$
H=K+U
$$

As a Kolmogorov model, (10) becomes

$$
\begin{aligned}
& \dot{\bar{\omega}}=\{\overline{\boldsymbol{\omega}}, K\}+\{\overline{\boldsymbol{\omega}}, U\}-\Lambda \overline{\boldsymbol{\omega}}+\mathbf{f} \\
& =\overline{\boldsymbol{\omega}} \times \nabla K+\overline{\boldsymbol{\omega}} \times \nabla U-\Lambda \overline{\boldsymbol{\omega}}+\mathbf{f} \\
& =\bar{\omega} \times \Pi \bar{\omega}+\bar{\omega} \times \beta-\Lambda \bar{\omega}+\mathbf{f} \\
& =\left[\begin{array}{c}
\frac{\left(I_{y}-I_{z}\right)}{\sqrt{I_{x} I_{y} I_{z}}} \bar{\omega}_{y} \bar{\omega}_{z} \\
\frac{\left(I_{z}-I_{x}\right)}{\sqrt{I_{x} I_{y} I_{z}}} \bar{\omega}_{x} \bar{\omega}_{z} \\
\frac{\left(I_{x}-I_{y}\right)}{\sqrt{I_{x} I_{y} I_{z}}} \bar{\omega}_{x} \bar{\omega}_{y}
\end{array}\right]+\left[\begin{array}{c}
\frac{h_{y}}{\sqrt{I_{x} I_{z}}} \bar{\omega}_{z}-\frac{h_{z}}{\sqrt{I_{x} I_{y}}} \bar{\omega}_{y} \\
\frac{h_{z}}{\sqrt{I_{x} I_{y}}} \bar{\omega}_{x} \\
-\frac{h_{y}}{\sqrt{I_{x} I_{z}}} \bar{\omega}_{x}
\end{array}\right] \\
& -\left[\begin{array}{c}
\frac{\mu_{x}}{I_{x}} \bar{\omega}_{x} \\
-\frac{\mu_{y}}{I_{y y}} \bar{\omega}_{y} \\
\frac{\mu_{z}}{I_{z}} \bar{\omega}_{z}
\end{array}\right]+\left[\begin{array}{c}
\frac{L_{x}}{\sqrt{I_{\mathrm{x}}}} \\
\frac{L_{y}}{\sqrt{I_{y}}} \\
\frac{L_{z}}{\sqrt{I_{z}}}
\end{array}\right],
\end{aligned}
$$

where

$$
\begin{gathered}
\boldsymbol{\Lambda}=\operatorname{diag}\left(\begin{array}{lll}
\frac{\mu_{x}}{I_{x}} & -\frac{\mu_{y}}{I_{y}} & \frac{\mu_{z}}{I_{z}}
\end{array}\right), \\
\mathbf{f}=\left[\begin{array}{lll}
\frac{L_{x}}{\sqrt{I_{x}}} & \frac{L_{y}}{\sqrt{I_{y}}} & \frac{L_{z}}{\sqrt{I_{z}}}
\end{array}\right]^{\mathrm{T}} .
\end{gathered}
$$

To study system (4) further, we rearrange (17). Substituting (9) into (17), we have

$$
\left[\begin{array}{c}
\sqrt{I_{x}} \dot{\omega}_{x} \\
\sqrt{I_{y}} \dot{\omega}_{y} \\
\sqrt{I_{z}} \dot{\omega}_{z}
\end{array}\right]=\left[\begin{array}{l}
\frac{\left(I_{y}-I_{z}\right)}{\sqrt{I_{x}}} \omega_{y} \omega_{z} \\
\frac{\left(I_{z}-I_{x}\right)}{\sqrt{I_{y}}} \omega_{x} \omega_{z} \\
\frac{\left(I_{x}-I_{y}\right)}{\sqrt{I_{z}}} \omega_{x} \omega_{y}
\end{array}\right]
$$

$$
+\left[\begin{array}{c}
\frac{h_{y}}{\sqrt{I_{x}}} \omega_{z}-\frac{h_{z}}{\sqrt{I_{x}}} \omega_{y} \\
\frac{h_{z}}{\sqrt{I_{y}}} \omega_{x} \\
-\frac{h_{y}}{\sqrt{I_{z}}} \omega_{x}
\end{array}\right]-\left[\begin{array}{c}
\frac{\mu_{x}}{\sqrt{I_{x}}} \omega_{x} \\
-\frac{\mu_{y}}{\sqrt{I_{y}}} \omega_{y} \\
\frac{\mu_{z}}{\sqrt{I_{z}}} \omega_{z}
\end{array}\right]
$$

$$
+\left[\begin{array}{c}
\frac{L_{x}}{\sqrt{I_{x}}} \\
\frac{L_{y}}{\sqrt{I_{y}}} \\
\frac{L_{z}}{\sqrt{I_{z}}}
\end{array}\right]
$$

Equation (19) is equivalent to (4).

Remark 1. Through the transformation and derivation of the Kolmogorov model (17), the total coupled torque of the original system (4) is decomposed into four torques: inertial, internal, dissipative, and external. The decomposition is convenient to uncover certain simple dynamic behavior caused by the simple torque; the comprehensive behavior arising from the combined torques is to be described in Section 5 .

\section{Energy of the Gyrostat System}

4.1. Energy Representation of the Gyrostat System. The energy decomposition is more important than the force decomposition in analyzing the dynamical behavior, because the force is a vector, whereas energy is a scalar. The former is difficult to analyze and to identify a relationship between force and definitive dynamic behavior of system, but the latter is relatively easier to quantify and therefore helpful in finding the mechanism underlying the dynamic behavior.

Normally, force and energy are in some form of coexistence. The four forms of energy (kinetic, potential, dissipated, and supplied) correspond, respectively, to the four forms of torques (inertial, internal, dissipative, and external) in the gyrostat system. There are internal energy exchanges between the kinetic and potential terms, and an internal-external energy exchange between the Hamiltonian and dissipated and supplied torques. Energy cycles take place among these 
four forms. However, the input energy is the external energy and the output energy is the dissipated energy for the system.

From (13) and (15), kinetic energy and potential energy have been given. Therefore, we need to derive a formula for the dissipated energy and supplied energy. Following [24], we set

$$
D=\frac{1}{2}\langle\overline{\boldsymbol{\omega}}, \Lambda \overline{\boldsymbol{\omega}}\rangle
$$

as the dissipative power, which is the changing rate of dissipated energy. From (9), we have

$$
D=\frac{1}{2}\langle\overline{\boldsymbol{\omega}}, \Lambda \overline{\boldsymbol{\omega}}\rangle=\frac{1}{2}\left(\mu_{x} \omega_{x}^{2}-\mu_{y} \omega_{y}^{2}+\mu_{z} \omega_{z}^{2}\right) .
$$

Terms $\mu_{x} \omega_{x}^{2},-\mu_{y} \omega_{y}^{2}$, and $\mu_{z} \omega_{z}^{2}$ are the damping power of the gyrostat system. Therefore, $2 D=\mu_{x} \omega_{x}^{2}-\mu_{y} \omega_{y}^{2}+\mu_{z} \omega_{z}^{2}$ is really the total dissipative power. Likewise, we define

$$
G=\langle\overline{\boldsymbol{w}}, \mathbf{f}\rangle=L_{x} \omega_{x}+L_{y} \omega_{y}+L_{z} \omega_{z},
$$

as the external power. Then the Kolmogorov model (17) is rewritten as

$$
\dot{\overline{\boldsymbol{\omega}}}=\{\overline{\boldsymbol{\omega}}, H\}-\nabla D+\nabla G .
$$

Remark 2. Differing from the original Kolmogorov model (5), in which only inertial torque and internal torque link with the kinetic and potential energies, the dissipative torque and external torque are expressed using the corresponding energies in (23).

We already have the external torque $\mathbf{f}$ and dissipative torque $\Lambda \overline{\boldsymbol{\omega}}$. Setting

$$
\mathbf{e}=\mathbf{f}-\Lambda \overline{\boldsymbol{w}},
$$

as the error torque, as given in [25], we define and expand

$$
E_{P}=\langle\overline{\boldsymbol{\omega}}, \mathbf{e}\rangle=\langle\overline{\boldsymbol{\omega}}, \mathbf{f}\rangle-\langle\overline{\boldsymbol{\omega}}, \boldsymbol{\Lambda} \overline{\boldsymbol{\omega}}\rangle=G-2 D,
$$

which gives the error power as the difference between the external power and total dissipative power.

\subsection{Energy Transformation. From (6), we have}

$$
\begin{aligned}
\langle\nabla K,\{\overline{\boldsymbol{\omega}}, H\}\rangle & =\langle\nabla K, \overline{\boldsymbol{\omega}} \times \nabla H\rangle=-\langle\overline{\boldsymbol{\omega}}, \nabla K \times \nabla H\rangle \\
& =\{K, H\} .
\end{aligned}
$$

Next, we have

$$
\begin{aligned}
& \frac{\partial K}{\partial \overline{\boldsymbol{\omega}}}\{\overline{\boldsymbol{\omega}}, H\}=\{K, H\}, \\
& \frac{\partial U}{\partial \overline{\boldsymbol{\omega}}}\{\overline{\boldsymbol{\omega}}, H\}=\langle U, H\rangle .
\end{aligned}
$$

From (13), (23), (24), and (27), we then have

$$
\begin{aligned}
\dot{K}= & \frac{\partial K}{\partial \overline{\boldsymbol{\omega}}} \dot{\overline{\boldsymbol{\omega}}}=\frac{\partial K}{\partial \overline{\boldsymbol{\omega}}}\{\overline{\boldsymbol{\omega}}, H\}+\langle\nabla K, \mathbf{e}\rangle \\
= & \{K, K\}+\{K, U\}+\langle\Pi \overline{\boldsymbol{\omega}}, \mathbf{e}\rangle \\
= & h_{y} \omega_{z} \omega_{x}\left(\Pi_{1}-\Pi_{3}\right)+h_{z} \omega_{y} \omega_{x}\left(\Pi_{2}-\Pi_{1}\right) \\
& -\left(\Pi_{1} \mu_{x} \omega_{x}^{2}-\Pi_{2} \mu_{y} \omega_{y}^{2}+\Pi_{3} \mu_{z} \omega_{z}^{2}\right) \\
& +\left(\Pi_{1} L_{x} \omega_{x}+\Pi_{2} L_{y} \omega_{y}+\Pi_{3} L_{z} \omega_{z}\right) .
\end{aligned}
$$

Therefore, the rate of change of kinetic energy is the sum of the exchange between the inside conservative part (kinetic and potential) and the exchange between kinetic and error energy. Likewise, we have

$$
\begin{aligned}
\dot{U}= & \frac{\partial U}{\partial \overline{\boldsymbol{\omega}}} \dot{\overline{\boldsymbol{\omega}}}=\frac{\partial U}{\partial \overline{\boldsymbol{\omega}}}\{\overline{\boldsymbol{\omega}}, H\}+\langle\nabla U, \mathbf{e}\rangle \\
= & -\{K, U\}+\langle\boldsymbol{\beta}, \mathbf{e}\rangle \\
= & h_{y} \omega_{z} \omega_{x}\left(\Pi_{3}-\Pi_{1}\right)+h_{z} \omega_{y} \omega_{x}\left(\Pi_{1}-\Pi_{2}\right) \\
& -\frac{h_{y} \mu_{y}}{\sqrt{I_{x} I_{y} I_{z}}} \omega_{y}+\frac{h_{z} \mu_{z}}{\sqrt{I_{x} I_{y} I_{z}}} \omega_{z}-\frac{h_{y} L_{y}}{\sqrt{I_{x} I_{y} I_{z}}} \\
& -\frac{h_{z} L_{z}}{\sqrt{I_{x} I_{y} I_{z}}} .
\end{aligned}
$$

Hence, the rate of change of potential energy is the negative exchange power between kinetic energy and potential energy and the exchange power between potential energy and error energy. For the Hamiltonian energy, we have

$$
\begin{aligned}
\dot{H} & =\langle\nabla H, \dot{\overline{\boldsymbol{\omega}}}\rangle=\{H, H\}+\langle\nabla H, \mathbf{e}\rangle \\
& =\langle\Pi \overline{\boldsymbol{\omega}}, \mathbf{e}\rangle+\langle\boldsymbol{\beta}, \mathbf{e}\rangle .
\end{aligned}
$$

Therefore, the rate of change in the Hamiltonian energy is determined by the exchange power between Hamiltonian energy and error energy.

4.3. Casimir Function and Error Power. The Casimir function $C$, like enstrophy or the potential vorticity in a fluid dynamical context, is very useful in analyzing stability conditions and the global description of a dynamical system [34]. Given that the Casimir energy function is $C(\mathbf{x})=(1 / 2)\|\mathbf{x}\|^{2}$, then $\nabla C(\mathbf{x})=\mathbf{x}$ and, for any $F \in f\left(R^{3}\right)$, we have

$$
\begin{aligned}
\{C, F\}(\mathbf{x}) & =-\mathbf{x} \cdot(\nabla C \times \nabla F)=-\mathbf{x} \cdot(\mathbf{x} \times \nabla F) \\
& =-\nabla F \cdot(\mathbf{x} \times \mathbf{x})=0 .
\end{aligned}
$$

Therefore, $C$ is a constant of the motion of the Hamiltonian system. Analogously, define the Casimir energy function for the gyrostat chaotic system as

$$
C=\frac{1}{2}\langle\overline{\boldsymbol{\omega}}, \overline{\boldsymbol{\omega}}\rangle=\frac{1}{2}\left(I_{x} \omega_{x}^{2}+I_{y} \omega_{y}^{2}+I_{z} \omega_{z}^{2}\right) .
$$


Remark 3. For a mechanical gyrostat system, the physical meanings of terms $I_{x} \omega_{x}^{2}, I_{y} \omega_{y}^{2}$, and $I_{z} \omega_{z}^{2}$ in (32) are stored mechanical rotational energies. Therefore, the Casimir energy is the total stored energy for the gyrostat system.

Calling the rate of change of the Casimir energy the Casimir power, then from (6), (24), and (31), the Casimir power is

$$
\begin{aligned}
\dot{C} & =\frac{\partial C}{\partial \overline{\boldsymbol{\omega}}}\{\overline{\boldsymbol{\omega}}, H\}+\langle\nabla C, \mathbf{e}\rangle=\{C, H\}+\langle\nabla C, \mathbf{e}\rangle \\
& =\langle\nabla C, \mathbf{e}\rangle=\langle\overline{\boldsymbol{\omega}}, \mathbf{e}\rangle \\
& =L_{x} \omega_{x}+L_{y} \omega_{y}+L_{z} \omega_{z}-\left(\mu_{x} \omega_{x}^{2}-\mu_{y} \omega_{y}^{2}+\mu_{z} \omega_{z}^{2}\right) .
\end{aligned}
$$

From (25), we obtain

$$
\dot{C}=E_{P}
$$

From (32) and (34), we have

$$
C(t)=\int_{0}^{t} E_{P}(\tau) d \tau+C_{0}
$$

Remark 4. The Casimir energy is the stored mechanical energy, but the Casimir power is the error power between the external and the dissipative.

Based on the conclusion concerning Casimir power [24, $25,31]$, we summarize as follows.

Remark 5. The Casimir power sign, sign switch, and the extension of the power switch are important indicators of the definitive dynamical behavior. The leap in Casimir energy level is also an important indicator. Both the switch in Casimir power sign and the Casimir energy leap influence the dynamical bifurcation. We conclude the following:

(1) If $\dot{C}(\mathbf{x})>0$ holds for all times, the supplied power is greater than the dissipative power, and the Casimir energy keeps increasing, and the system diverges as a source.

(2) If $\dot{C}(\mathbf{x})<0$ holds for all times, then the orbit of the system converges to a sink.

(3) A nonzero constant Casimir function, i.e., $\dot{C} \equiv 0$ with $C \neq 0$, signifies a conservative system that produces a periodic orbit.

(4) If $\dot{C}(\mathbf{x})$ converges and finally leaps periodically along the zero line, then the orbit is a limit cycle.

(5) If $\dot{C}(\mathbf{x})$ is bounded and irregularly vibrating around the zero line, then the orbit of the system is either chaotic or aperiodic.

(6) If $\dot{C}(\mathbf{x})$ converges into a zero asymptotically, the orbit converges to a sink.

(7) The bifurcation of Casimir power is identical with the bifurcation of the system's states or the spectrum of the Lypapunov exponent. However, the former is analytic, which is helpful in revealing the mechanism of production of different dynamical behaviors.
The extremal points of the Casimir energy for the gyrostat chaotic system (4) are obtained by letting $\dot{C}=0$. They fall on a triaxle hyperboloid,

$$
\begin{aligned}
& \Xi_{0}: \mu_{x}\left(\omega_{x}-\frac{L_{x}}{2 \mu_{x}}\right)^{2}-\mu_{y}\left(\omega_{y}+\frac{L_{y}}{2 \mu_{y}}\right)^{2} \\
& +\mu_{z}\left(\omega_{z}-\frac{L_{z}}{2 \mu_{z}}\right)^{2}=\frac{L_{x}^{2}}{4 \mu_{x}}-\frac{L_{y}^{2}}{4 \mu_{y}}+\frac{L_{z}^{2}}{4 \mu_{z}} .
\end{aligned}
$$

\section{Dynamical Behaviors Influenced by Force Field and Energy}

We now investigate the dynamic behavior corresponding to the different forms of torques in the gyrostat system (4) to find out the effects of each type of torque and energy on the system. We take $I_{x}=0.85 \mathrm{Kg} \cdot \mathrm{m}^{2}, I_{y}=0.45 \mathrm{Kg} \cdot \mathrm{m}^{2}$, $I_{z}=0.2 \mathrm{Kg} \cdot \mathrm{m}^{2}, h_{y}=0.57416 \mathrm{Kg} \cdot \mathrm{m}^{2} / \mathrm{s}, h_{z}=2.38 \mathrm{Kg} \cdot \mathrm{m}^{2} / \mathrm{s}$, $\mu_{x}=7.6$ N.m.s, $\mu_{y}=6.42$ N.m.s, and $\mu_{z}=5.8$ N.m.s and set the external torques $L_{x}=0$ N.m, $L_{y}=0$ N.m, and $L_{z}=$ 22.8 N.m, initial conditions $\omega_{x 0}=\omega_{z 0}=\omega_{y 0}=2.5 \mathrm{rad} / \mathrm{s}$, and sampling time $\tau=0.0001 \mathrm{~s}$.

5.1. Gyrostat System Governed by Inertia and Internal Force Field. System (17) contains the inertial torque and internal torque, i.e., it has kinetic energy and potential energy. Correspondingly, the system is written as

$$
\dot{\overline{\boldsymbol{\omega}}}=\{\overline{\boldsymbol{\omega}}, K\}+\{\overline{\boldsymbol{\omega}}, U\},
$$

which means system (4) becomes

$$
\left[\begin{array}{c}
I_{x} \dot{\omega}_{x} \\
I_{y} \dot{\omega}_{y} \\
I_{z} \dot{\omega}_{z}
\end{array}\right]=\left[\begin{array}{c}
\left(I_{y}-I_{z}\right) \omega_{y} \omega_{z} \\
\left(I_{z}-I_{x}\right) \omega_{x} \omega_{z} \\
\left(I_{x}-I_{y}\right) \omega_{x} \omega_{y}
\end{array}\right]+\left[\begin{array}{c}
h_{y} \omega_{z}-h_{z} \omega_{y} \\
h_{z} \omega_{x} \\
-h_{y} \omega_{x}
\end{array}\right] .
$$

The rotation of the rotor relative to the carrier frame produces angular momenta $h_{x}, h_{y}, h_{z}$ and corresponding potential energy, both of which have an effect on the dynamics of the system. The principal moments of inertia of the gyrostat system, $I_{x}, I_{y}, I_{z}$, determine the kinetic energy. A periodic orbit is generated [Figure 2(a)]. Why is the periodic orbit produced, and how do the two forms of energies influence the behavior? There is a transfer between kinetic energy and potential energy. From (28) and (29), we obtain

$$
\begin{aligned}
\dot{K} & =\{K, U\} \\
& =h_{y} \omega_{z} \omega_{x}\left(\Pi_{1}-\Pi_{3}\right)+h_{z} \omega_{y} \omega_{x}\left(\Pi_{2}-\Pi_{1}\right), \\
\dot{U} & =-\{K, U\} .
\end{aligned}
$$

Therefore, the amount of kinetic energy loss equals the potential energy gained, meaning the system is also conservative with

$$
\dot{H}=\{H, H\}=0 .
$$




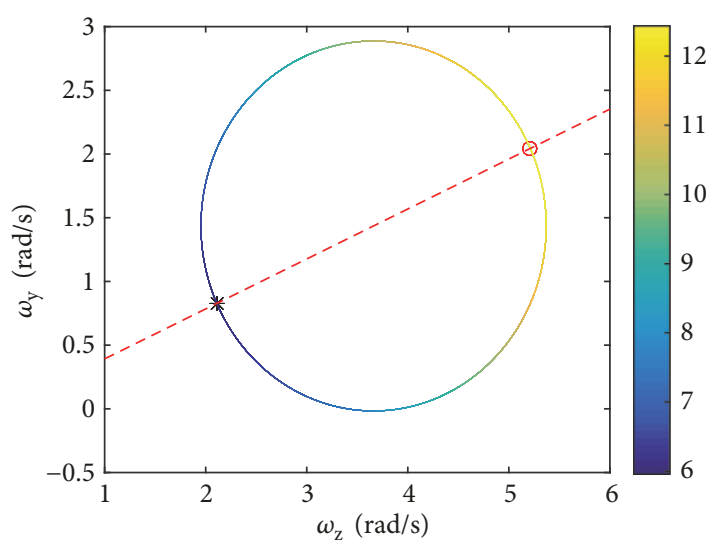

(a)
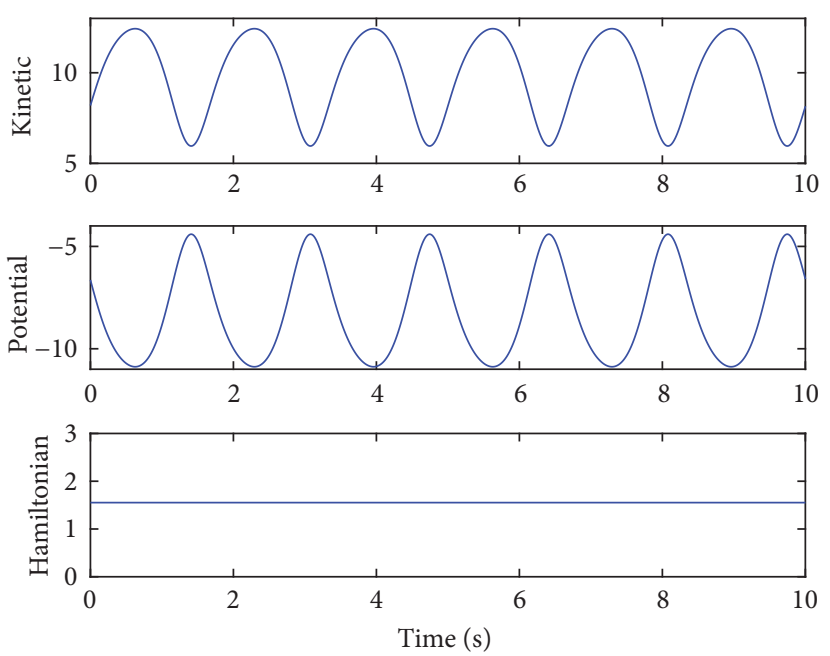

(b)

FIGURE 2: System governed by inertial and internal torques: (a) a periodic orbit with a kinetic energy cycle, (b) kinetic, potential, and Hamiltonian energy.

The exchange of kinetic energy and potential energy and the conservation of the total ensures the system behaves periodically. The periodic orbit is symmetrical along the red dotted line [Figure 2(a)]. The color coding indicates the kinetic energy value; a change from dark blue to yellow means the kinetic energy increases, $\dot{K}>0$. When the orbit reaches the red circle, the kinetic energy reaches its maximum. With $\dot{K}<0$, the kinetic energy decreases until reaching a minimum (black stars) upon which the cycle repeats. Figure 2(b) reflects the two forms of periodic energy cycling; also, the amount of kinetic energy loss equals the potential energy gained. The Hamiltonian is constant from (39) with $H=1.5522(\mathrm{~J})$. The Casimir energy is also constant with $C=C_{0}=4.6875(\mathrm{~J})$, which indicates that the system does not exchange Casimir energy with the outside.

Similarly, when the system contains only kinetic energy or potential energy, the system still undergoes a periodic orbit with energy conserved, but the two forms of orbits are simpler than the orbit produced by the sum of the two energies.

5.2. Gyrostat System Governed by Inertia, Internal, and Dissipative Torques. This governing equation of this gyrostat chaotic system is written as

$$
\dot{\overline{\boldsymbol{\omega}}}=\{\overline{\boldsymbol{\omega}}, K\}+\{\overline{\boldsymbol{\omega}}, U\}-\nabla D .
$$

The system contains kinetic, potential, and dissipative energies. System (4) becomes

$$
\begin{aligned}
{\left[\begin{array}{c}
I_{x} \dot{\omega}_{x} \\
I_{y} \dot{\omega}_{y} \\
I_{z} \dot{\omega}_{z}
\end{array}\right]=} & {\left[\begin{array}{c}
\left(I_{y}-I_{z}\right) \omega_{y} \omega_{z} \\
\left(I_{z}-I_{x}\right) \omega_{x} \omega_{z} \\
\left(I_{x}-I_{y}\right) \omega_{x} \omega_{y}
\end{array}\right]+\left[\begin{array}{c}
h_{y} \omega_{z}-h_{z} \omega_{y} \\
h_{z} \omega_{x} \\
-h_{y} \omega_{x}
\end{array}\right] } \\
& -\left[\begin{array}{c}
\mu_{x} \omega_{x} \\
-\mu_{y} \omega_{y} \\
\mu_{z} \omega_{z}
\end{array}\right] .
\end{aligned}
$$

The divergence of the system is

$$
\operatorname{div}(V)=\frac{\partial \dot{\omega}_{x}}{\partial \omega_{x}}+\frac{\partial \dot{\omega}_{y}}{\partial \omega_{y}}+\frac{\partial \dot{\omega}_{z}}{\partial \omega_{z}}=-\frac{\mu_{x}}{I_{x}}+\frac{\mu_{y}}{I_{y}}-\frac{\mu_{z}}{I_{z}},
$$

where $V$ is the volume in phase space of the system, with solution $V=V_{0} \exp \left(-\left(\mu_{x} / I_{x}-\mu_{y} / I_{y}+\mu_{z} / I_{z}\right)\right)$. With the given parameter values, we obtain $-\mu_{x} / I_{x}+\mu_{y} / I_{y}-\mu_{z} / I_{z}=$ -23.6745 , indicating the system is dissipative, so the volume index of the phase space decreases to 0 . In addition, the system is fractal for Lyapunov dimension $L_{\mathrm{d}}<3$.

There are two forms of dissipation: Rayleigh and nonRayleigh [23]. In physics, it frequently happens that the frictional (damping) force is proportional to the velocity, so that its $x$ component has the form $F_{x_{i}}=-k_{x_{i}} v_{x_{i}}$. When all the friction coefficients, $k_{x_{i}}$ are positive, the dissipation is Rayleigh, otherwise, non-Rayleigh. Therefore, system (42) is a non-Rayleigh dissipative system with $\partial \dot{\omega}_{y} / \partial \omega_{y}=\mu_{x} / I_{x}>0$; likewise, the original gyrostat system (4) is non-Rayleigh. A Rayleigh dissipative system without an external force never produces chaos for it evolves towards a sink [20]. System (42) is non-Rayleigh dissipative, with term $\mu_{y} \omega_{y}$ playing the role of a negative damping or external torque, so we cannot determine whether it produces a sink even despite having a negative divergence. However, we find the mechanism from the Casimir power, which is

$$
\dot{C}=-\mu_{x} \omega_{x}^{2}+\mu_{y} \omega_{y}^{2}-\mu_{z} \omega_{z}^{2} .
$$

From (44), the Casimir power has an uncertainty in terms of sign; probably, the Casimir power varies and oscillates with the system orbiting. This is confirmed by Figure 3(a). The oscillations of Casimir power reflect that the system has a sudden energy exchange between input and output. Although system (42) does not contain the external torque, it contains a negative damping term $\mu_{y} \omega_{y}$, which plays the role of an external torque. Actually, the system generates a four-wing 


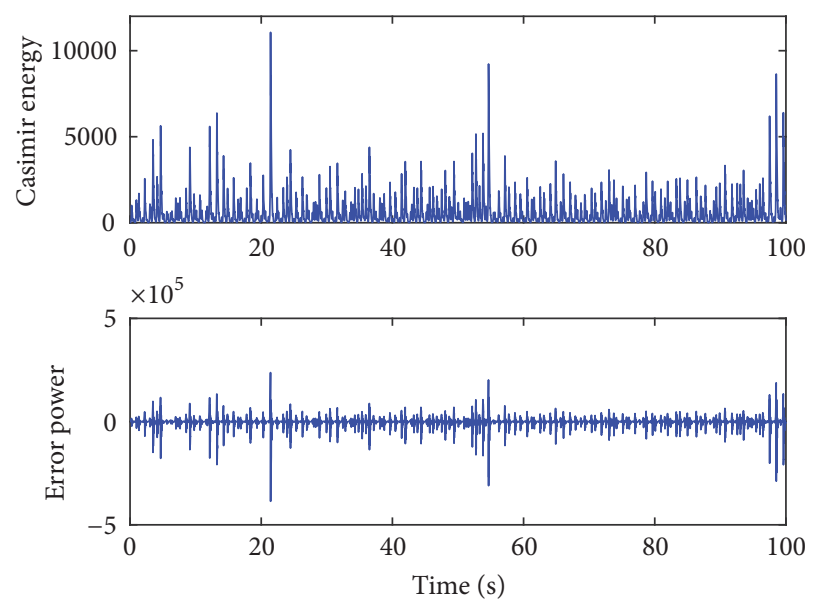

(a)

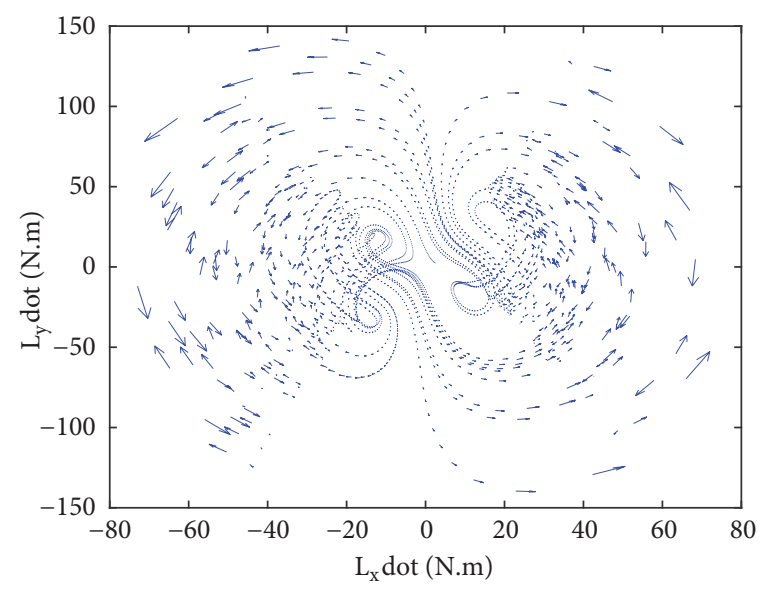

(c)

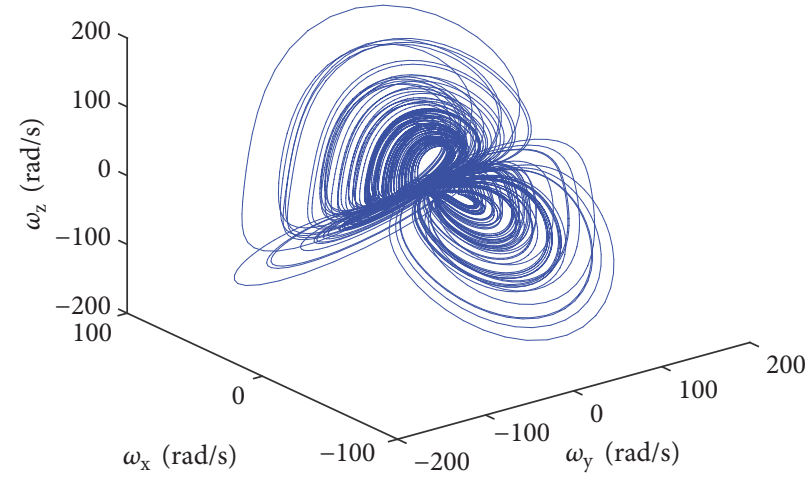

(b)

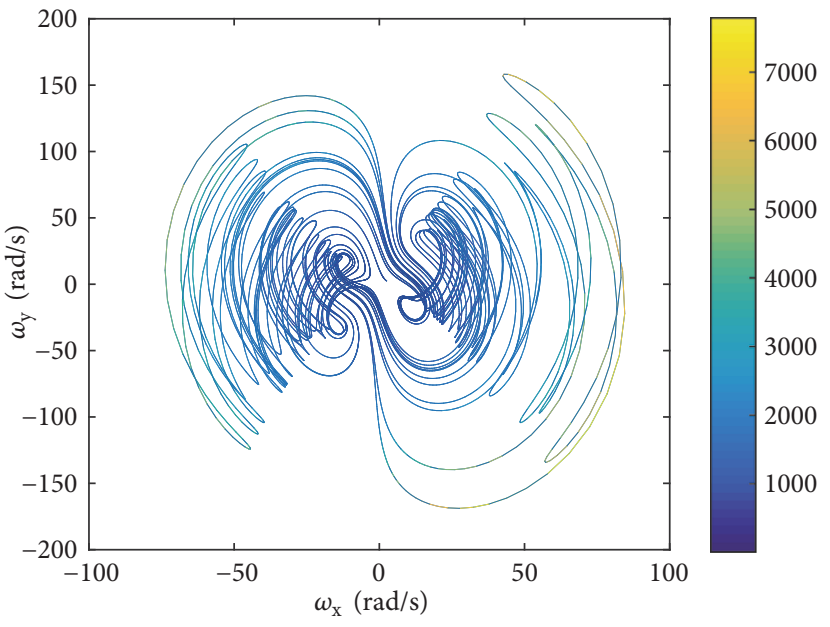

(d)

FIGURE 3: System governed by inertia, internal, and dissipative torques: (a) Casimir energy and Casimir power, (b) four-wing chaotic orbit, (c) phase plane of force field, and (d) phase plane orbit along with flowing Casimir energy.

chaotic attractor [Figure 3(b)] with Lyapunov exponents $L_{1}=$ 3.8719, $L_{2}=0, L_{3}=-27.5464$, and fractal Lyapunov dimension $L_{d}=2.1406<3$. Although the phase-space volume of the system converges to zero, the energy does not.

From (4), the force field is the vector field, which is also the reaction torque $\left[I_{x} \dot{\omega}_{x}, I_{\mathrm{y}} \dot{\omega}_{y}, I_{z} \dot{\omega}_{z}\right]^{\mathrm{T}}$, and because $L_{x}=I_{x} \omega_{x}, L_{y}=I_{y} \omega_{y}$, and $L_{z}=I_{z} \omega_{z}$, the force field vector is $\left[\dot{L}_{x}, \dot{L}_{y}, \dot{L}_{z}\right]^{\mathrm{T}}=\left[I_{x} \dot{\omega}_{x}, I_{y} \dot{\omega}_{y}, I_{z} \dot{\omega}_{z}\right]^{\mathrm{T}}$. We can plot the force field to show how the force impacts the orbit of system. Figure 3 (c) presents the reaction torque in $\dot{L}_{x}-\dot{L}_{y}$ plane, which is the force field flow of the system. Figure 3(d) shows a trajectory phase diagram along with flowing Casimir energy in which the stored energy changes from a minimum to a maximum and continually cycles. Compared with Figure 3(d), the magnitude of the torque in the torque field determines the amount of energy stored in the orbit, and the direction of the orbit is also controlled by the direction of the torque [Figure 3(c)].
5.3. Gyrostat System Governed by All Four Force Fields. When the gyrostat system contains all four torques-inertial, internal, dissipative (disturbance torques), and external-the governing equation is

$$
\dot{\overline{\boldsymbol{\omega}}}=\{\overline{\boldsymbol{\omega}}, H\}-\nabla D+\nabla G,
$$

which is fully equivalent to system (4). The Lyapunov exponents are found to be $L_{1}=3.8975, L_{2}=0, L_{3}=-27.572$, and the Lyapunov dimension $L_{d}=2.1411<3$, when the external torque is set to $L_{x}=0, L_{y}=0, L_{z}=22.18$. Comparing system (42) with system (4), we find that the positive Lyapunov exponent $L_{1}=3.8975$ is larger than $L_{1}=$ 3.8719 for a system without external torque. Additionally, the Lyapunov dimension $L_{d}=2.1411$ is slightly bigger compared with the value $L_{d}=2.1406$ of the system (42). Therefore, the existence of external torque complicates the dynamics and chaos of the gyrostat system [Figure 4(a)]. Note that external torque does not always make the system more chaotic or disordered, but it does add to the complexity of the system. 


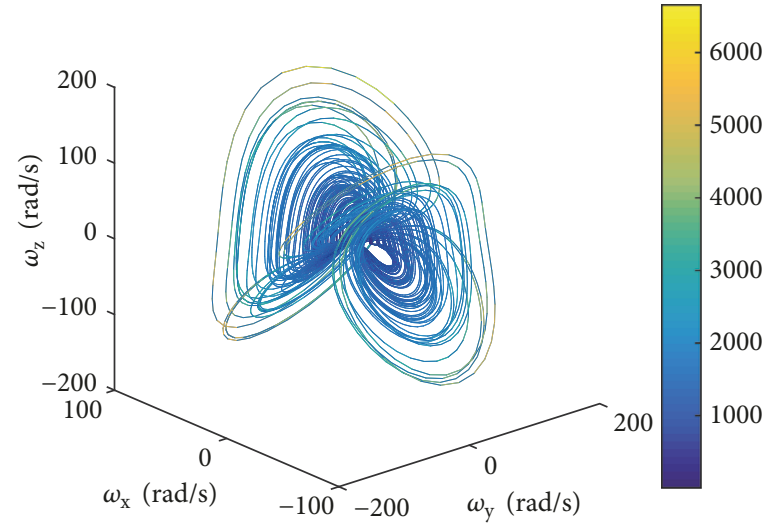

(a)

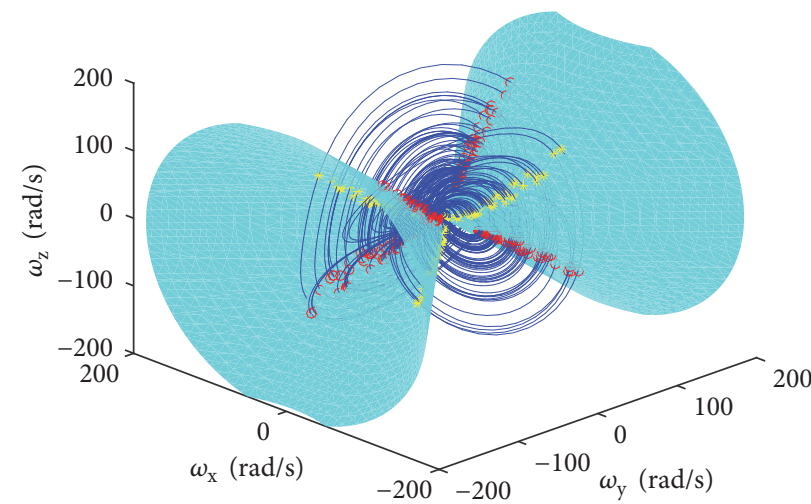

(c)
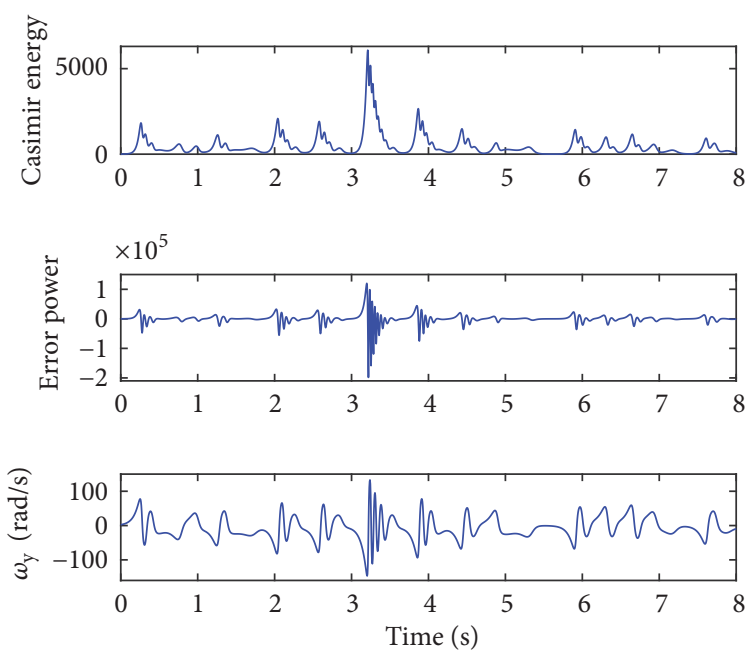

(b)

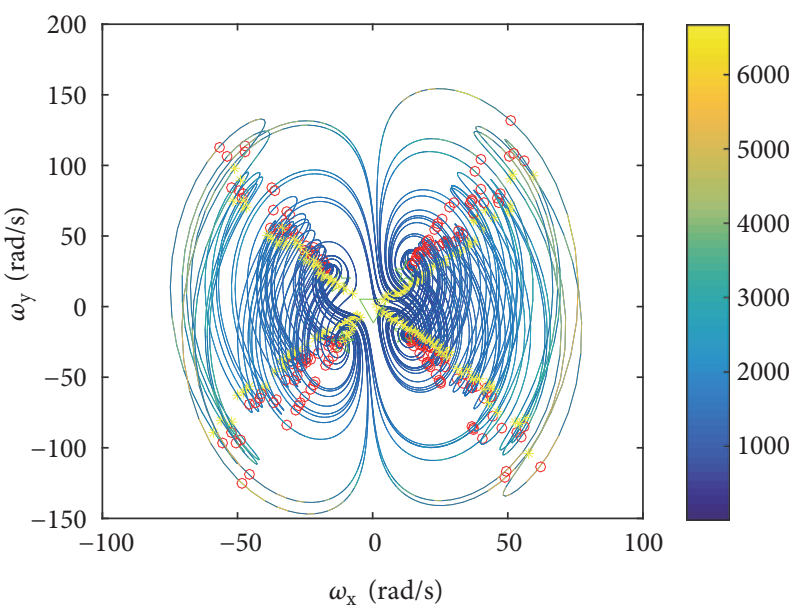

(d)

FIGURE 4: System under full torques. (a) 3D orbits with flowing stored energy, (b) time series of the stored energy, error power, and angular velocity $\omega_{x}$, (c) intersection of the chaotic system trajectory and hyperboloid of the Casimir function, the maxima and minima of which are marked by red circles and yellow stars, respectively, and (d) signs of extrema and equilibrium points on the four-wing chaotic attractor.

Figure 4(a) shows that the value of the Casimir energy (stored energy) varies with the orbit of the system; that is, the stored energy changes from a minimum to a maximum value and circulates constantly. From (33) and (34), the Casimir power (error power) is

$$
\begin{aligned}
\dot{C} & =E_{P} \\
& =L_{x} \omega_{x}+L_{y} \omega_{y}+L_{z} \omega_{z}-\left(\mu_{x} \omega_{x}^{2}-\mu_{y} \omega_{y}^{2}+\mu_{z} \omega_{z}^{2}\right) .
\end{aligned}
$$

In the middle panel of Figure 4(b), we obtain an error power between the dissipation and external energy that shifts in the range $\left[-1.974 \times 10^{-5}, 1.185 \times 10^{5}\right]$. The energy stored in the system is released and absorbed rapidly, causing the orbit of the system to become chaotic and folded [see top and bottom panels in Figure 4(b)].
The Casimir power is given by (33), and the intersected hyperboloid with chaotic attractor is given by $\Xi_{0}$ in (36). The intersected hyperboloid $\Xi_{0}$ is rewritten as

$$
\begin{aligned}
\Xi_{0}: & \mu_{x}\left(\omega_{x}-\frac{L_{x}}{2 \mu_{x}}\right)^{2}-\mu_{y}\left(\omega_{y}-\frac{L_{y}}{2 \mu_{y}}\right)^{2} \\
& +\mu_{z}\left(\omega_{z}-\frac{L_{z}}{2 \mu_{z}}\right)^{2}=\frac{L_{x}^{2}}{4 \mu_{x}}-\frac{L_{y}^{2}}{4 \mu_{y}}+\frac{L_{z}^{2}}{4 \mu_{z}} .
\end{aligned}
$$

It is designed to analyze the changes in Casimir energy in the system trajectory [Figure 4(c)], from which we may find the maxima and minima of the Casimir energy (32). The orbit of the chaotic attractor of system (4) intersects the hyperboloid with a change in Casimir energy [Figure 4(c)]. When $\dot{C}>$ 0 , i.e., the orbits are located inside the hyperboloid, the Casimir energy keeps increasing until it reaches a maximum 
TABLE 1: Equilibrium points and corresponding eigenvalues of system (4).

\begin{tabular}{lccr}
\hline Equilibrium point & \multicolumn{3}{c}{ Eigenvalue $\left(\lambda_{1}, \lambda_{2}, \lambda_{3}\right)$} \\
\hline$S_{1}(11.6173,-17.9707,-11.6170)$ & -33.2194 & $4.7725+\mathrm{j} 18.9858$ & $4.7725-\mathrm{j} 18.9858$ \\
$S_{2}(-12.5942,17.9807,-10.4397)$ & -32.9280 & $4.6267+\mathrm{j} 19.5380$ & $4.6267-\mathrm{j} 19.5380$ \\
$S_{3}(0.2935,0.0071,3.9021)$ & -9.0583 & -28.8997 & 14.2835 \\
$S_{4}(12.2571,23.8915,22.9136)$ & -37.6678 & $6.9966+\mathrm{j} 19.8438$ & $6.9966-\mathrm{j} 19.8438$ \\
$S_{5}(-11.5737,-25.6311,25.5351)$ & -38.9770 & $7.6512+\mathrm{j} 20.4982$ & $7.6512-\mathrm{j} 20.4982$ \\
\hline
\end{tabular}

(red circle). Conversely, when $\dot{C}<0$, i.e., the orbits are located outside the hyperboloid, the Casimir energy starts to decrease, eventually to a minimum (yellow stars), where the cycle then repeats. Figure $4(\mathrm{~d})$ presents a projection of the chaotic attractor onto the $\omega_{x}-\omega_{y}$ plane with the Casimir energy (color coded) and signs: maxima (red circles) and minima (yellow stars). The analysis above demonstrates that the Casimir energy and its associated power are good indicators in understanding the folding and expanding of the orbit of system.

The equilibrium point of system (4) and its corresponding eigenvalues are presented in Table 1 . Point $S_{3}$ is the saddle node, and the remaining equilibrium points are saddle focus nodes. The five equilibrium points are on the surface of $\Xi_{0}$ [Figure 4(d)], with point $S_{3}$ marked by a green triangle, and $S_{1}, S_{2}, S_{4}$, and $S_{5}$ marked by the four green pentagonal stars on the subcentral position of the four-wing chaotic attractor. We find that the four saddle focus nodes [Figure 4(d)] are also connection points of the maxima (red circles) and minima (yellow stars) of stored energy.

\section{Bifurcation and Dynamics Analysis of a Gyrostat System}

In the dynamic analysis of the gyrostat system, changes in system parameters affect the dynamics of the system. Because some parameters are determined by their structure, these system parameters are fixed at $I_{x}=0.85 \mathrm{Kg} \cdot \mathrm{m}^{2}, I_{y}=$ $0.45 \mathrm{Kg} \cdot \mathrm{m}^{2}, I_{z}=0.2 \mathrm{Kg} \cdot \mathrm{m}^{2}, h_{y}=0.57416 \mathrm{Kg} \cdot \mathrm{m}^{2} / \mathrm{s}, h_{z}=$ $2.38 \mathrm{Kg} \cdot \mathrm{m}^{2} / \mathrm{s}$.

6.1. Dynamics Analysis of Gyrostat System by Varying Parameter. Our purpose is to study the effects of the dissipative damping parameters on the dynamic behavior of the system in the gyrostat system with full torques, i.e., system (4). In this section, we demonstrate the similarity between the bifurcation diagram of the state and the bifurcation of the Casimir power highlighting that the Casimir power is a good indicator in analyzing the dynamics of the system.

Figure 5(a) demonstrates the state bifurcation diagram of the gyrostat system with respect to damping parameter $\mu_{x} \in[1.2,13.4]$. The system undergoes rich bifurcation dynamics with sinks, periodic orbits, multiperiodic orbits, and chaos, and then the reverse process. When the gyrostat system operates in the chaotic state, the angular velocity oscillates tremendously and, suddenly, there occurs a motion which would probably disrupt the system in real situations.
Therefore, the dissipative term or disturbance affects the system hugely. Other damping factors and other parameters most assuredly influence system dynamics but are not studied in the paper. Figure 5(a1) amplifies the state bifurcation in the range $\mu_{x} \in[1.2,2.5]$, in which the bifurcation process is clearer. The Lyapunov exponent spectrum with respect to the same parameter $\mu_{x} \in[1.2,13.4]$ reflecting the bifurcation process is exactly the same as the state bifurcation diagram [Figure 5(a)]; Figure 5(b1) is contrasted with Figure 5(a1).

What makes the bifurcation take place? We are unable to find a clue from the state bifurcation diagram and not from the Lyapunov exponent spectrum. However, we may find a clue from the Casimir power, (33) and (34), which are rewritten as

$$
\begin{aligned}
\dot{C} & =E_{p} \\
& =L_{x} \omega_{x}+L_{y} \omega_{y}+L_{z} \omega_{z}-\left(\mu_{x} \omega_{x}^{2}-\mu_{y} \omega_{y}^{2}+\mu_{z} \omega_{z}^{2}\right) .
\end{aligned}
$$

The Casimir power reflects the error power between the supplied and the dissipated power. When the damping parameter $\mu_{x}$ is varied, the sign of the Casimir power changes; therefore, the pattern by which the system absorbs and expends energy always switches, resulting thereby in the bifurcation behavior. Similarly, varying other parameters impacts the bifurcation of the gyrostat system.

The bifurcations of the Casimir power with respect to parameter $\mu_{x}$ are shown in Figures 5(c) and 5(c1). As parameter $\mu_{x}$ varies, the sign of Casimir power switches. The positive (negative) implies the supplied power is greater (less) than the dissipative power, so the Casimir energy increases (decreases) [Figures 5(d) and 5(d1)]. When the Casimir energy level leaps from one level to another, a bifurcation of the definitive behavior of the system takes place. When the Casimir power converges to zero in the range $\mu_{x} \in[1.2,1.39]$, the system orbit converges to a sink [Figure $5(\mathrm{cl})$ ], which is identical to the range of the sink in Figures 5(a1), 5(b1), and 5(d1) in accord with Remark 5(6). When the Casimir power bifurcates from zero to switching periodically along the zero line in the range $\mu_{x} \in(1.39,2.04]$, the Casimir energy leaps when $\mu_{x}=$ 1.39 at which the system's definitive behavior bifurcates to a periodic orbit in accord with Remark 5(4). As the switching amplitude of the Casimir power reaches some threshold, i.e., the Casimir energy leaps to a new level at $\mu_{x}=2.04$, the system behavior loses its stable periodicity and transits to the next definitive behavior, i.e., periodic-doubling. At point $\mu_{x}=2.205$, the Casimir energy leaps to a new level, the system orbit loses stable periodic multidoubling behavior and transits to an irregular oscillation, i.e., chaos or a chaotic 


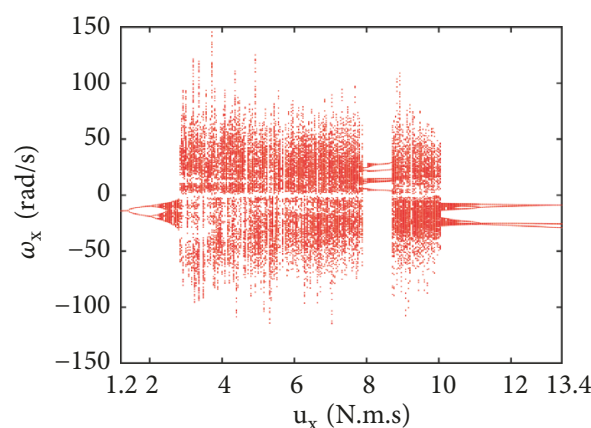

(a)

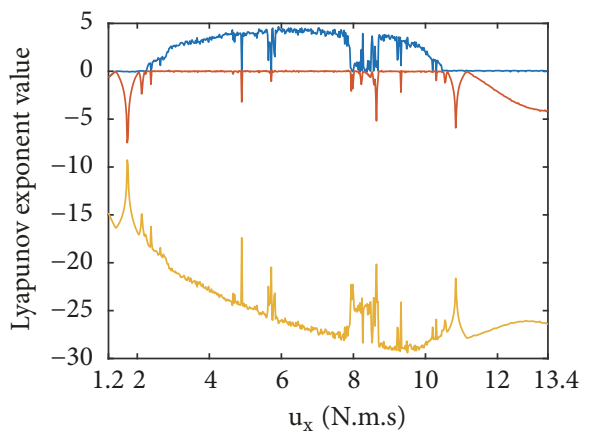

$-\mathrm{L}_{1}$
$-\mathrm{L}_{2}$
$\mathrm{~L}_{3}$

(b)

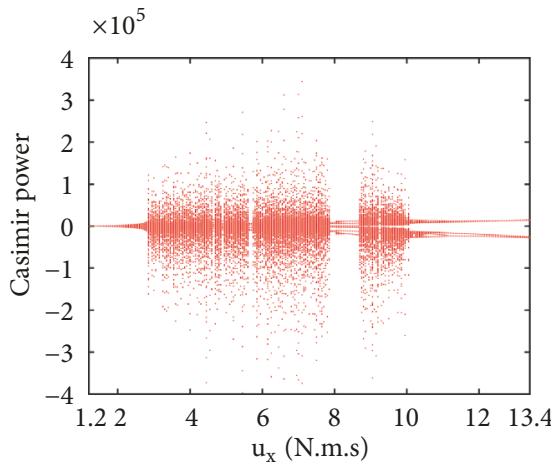

(c)

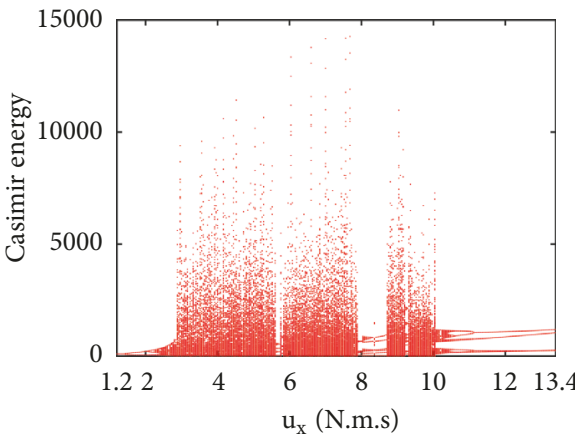

(d)

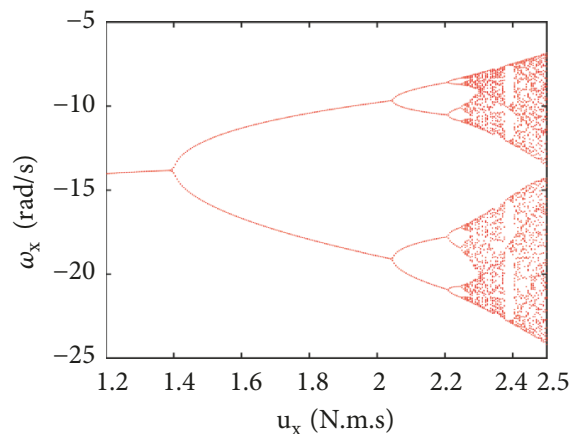

(a1)

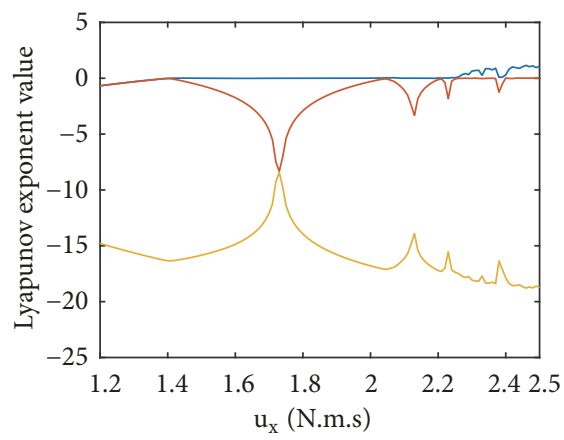

- $\mathrm{L}_{1}$

$-\mathrm{L}_{3}$

(b1)

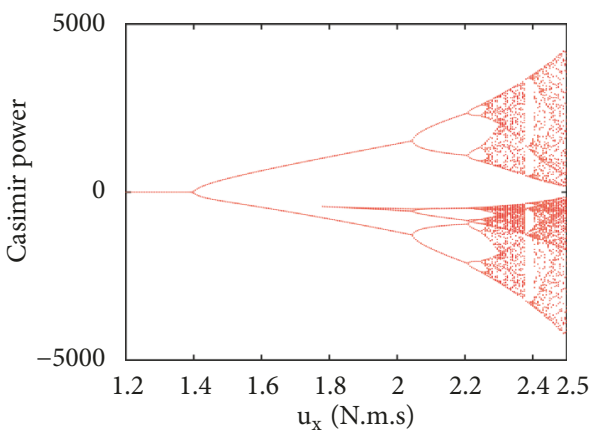

(c1)

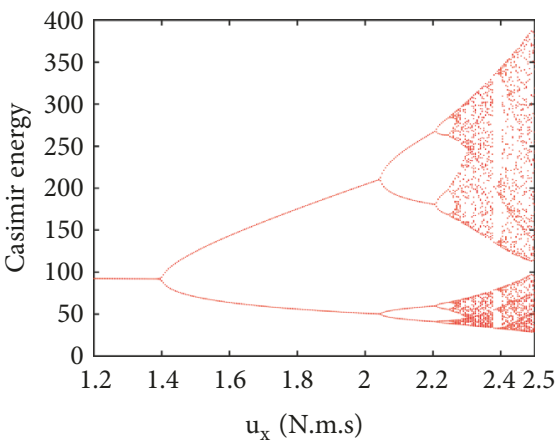

(d1)

FIGURE 5: Bifurcation of the gyrostat system (4) with respect to $\mu_{x}$ with $\mu_{y}=6.42 \mathrm{~N} . \mathrm{m} . \mathrm{s}, \mu_{z}=5.8 \mathrm{~N} \cdot \mathrm{m} . \mathrm{s}, L_{x}=L_{y}=0, L_{z}=22.8 \mathrm{~N} . \mathrm{m},(\mathrm{a})$ bifurcation diagram of state $\omega_{x}$, (b) Lyapunov exponent spectrum, (c) bifurcation of Casimir power, and (d) bifurcation of Casimir energy. 
attractor is constructed in accordance with Remark 5(6). The violent absorption and release of energy stored in the system causes the generation of chaos.

\section{Conclusion}

A chaotic gyrostat model was established that provides a reference model for researchers engaged in dynamical and gyrostat studies. The gyrostat was transformed into a Kolmogorov model to separate the torques. The conversion of energy and the cycles performed were revealed through identifying four forms of energy-kinetic, potential, dissipative, and supplied. The Casimir power is equivalent to the error power between the supplied power and the dissipative power. The Casimir power and energy leap were successfully used as indicators that assisted in evaluating the different dynamical features, such as sinks, periodic orbits, and chaos, and were identical to the indicators of the Lyapunov exponent spectrum and state bifurcation. However, the Casimir power is more convenient for the analysis of the mechanism in understanding different types of dynamics generation because of the analytical form. The idea and method may be applicable to the analyses of mechanisms for other dynamical systems. Therefore, how to find some direction information from the form of Casimir power prior to the numerical simulation is a significant work. Surely, there are many further investigations, such as Hopf bifurcation, pitchfork bifurcation, and visual demonstration, and hidden attractor can be conducted, which has provided some open topics for the other academic researchers.

\section{Data Availability}

The data used to support the findings of this study can be computed by the relative equations and parameters given in the article using ODE5 solver in Matlab and are available from the corresponding author upon request.

\section{Conflicts of Interest}

The authors declare that they have no conflicts of interest.

\section{Acknowledgments}

This work is supported by the National Natural Science Foundation of China (61873186) and Natural Science Foundation of Tianjin City (17JCZDJC38300). We thank Richard Haase, Ph.D., from Liwen Bianji, Edanz Group China (www.liwenbianji.cn/ac), for editing the English text of a draft of this manuscript.

\section{References}

[1] J. Wittenburg, Dynamics of Systems of Rigid Bodies, Teubner, 1977.

[2] P. J. Holmes and J. E. Marsden, "Horseshoes in perturbations of Hamiltonian systems with two degrees of freedom," Communications in Mathematical Physics, vol. 82, no. 4, pp. 523-544, 1982.
[3] P. J. Holmes and J. E. Marsden, "Horseshoe and Arnold diffusion for Hamiltonian system on Lie groups," Indiana University Mathematics Journal, vol. 32, no. 2, pp. 273-309, 1983.

[4] P. Krishnaprasad, "Lie-Poisson structures, dual-spin spacecraft and asymptotic stability," Nonlinear Analysis: Theory, Methods \& Applications, vol. 9, no. 10, pp. 1011-1035, 1985.

[5] X. Tong, B. Tabarrok, and F. P. Rimrott, "Chaotic motion of an asymmetric gyrostat in the gravitational field," International Journal of Non-Linear Mechanics, vol. 30, no. 3, pp. 191-203, 1995.

[6] X. Tong and B. Tabarrok, "Melnikov's method for rigid bodies subject to small perturbation torques," Archive of Applied Mechanics, vol. 66, no. 4, pp. 215-230, 1996.

[7] Y. Z. Liu and F. P. J. Rimrott, "Global motion of a dissipative asymmetric gyrostat," Archive of Applied Mechanics, vol. 62, no. 5, pp. 329-337, 1992.

[8] J. Kuang, S. Tan, and A. Y. T. Leung, "Chaotic attitude motion of satellites under small perturbation torques," Journal of Sound and Vibration, vol. 235, no. 2, pp. 175-200, 2000.

[9] J. Kuang, S. Tan, K. Arichandran, and A. Y. T. Leung, "Chaotic dynamics of an asymmetrical gyrostat," International Journal of Non-Linear Mechanics, vol. 36, no. 8, pp. 1213-1233, 2001.

[10] J. Kuang, S. Tan, K. Arichandran, and A. Y. T. Leung, "Chaotic attitude motion of gyrostat satellite via Melnikov method," International Journal of Bifurcation and Chaos, vol. 11, no. 05, pp. 1233-1260, 2001.

[11] M. Iñarrea, "Chaotic pitch motion of a magnetic spacecraft with viscous drag in an elliptical polar orbit," International Journal of Bifurcation and Chaos, vol. 21, no. 07, pp. 1959-1975, 2011.

[12] J. Kuang, S. Tan, and A. Y. Leung, “On Melnikov's method in the study of chaotic motions of gyrostat," International Journal of Control, vol. 75, no. 5, pp. 328-351, 2002.

[13] V. S. Aslanov and A. V. Doroshin, "Chaotic dynamics of an unbalanced gyrostat," Journal of Applied Mathematics and Mechanics, vol. 74, no. 5, pp. 524-535, 2010.

[14] V. S. Aslanov and V. V. Yudintsev, "Dynamics and chaos control of asymmetric gyrostat satellites," Cosmic Research, vol. 52, no. 3, pp. 216-228, 2014.

[15] W. Zhang, J. Zhang, and M. Yao, "The extended Melnikov method for non-autonomous nonlinear dynamical systems and application to multi-pulse chaotic dynamics of a buckled thin plate," Nonlinear Analysis: Real World Applications, vol. 11, no. 3, pp. 1442-1457, 2010.

[16] J. Zhang and W. Zhang, "An extended high-dimensional Melnikov analysis for global and chaotic dynamics of a nonautonomous rectangular buckled thin plate," Science China Physics, Mechanics and Astronomy, vol. 55, no. 9, pp. 1679-1690, 2012.

[17] V. I. Arnold, "Kolmogorov's hydrodynamic attractors," Proceedings of the Royal Society A Mathematical, Physical and Engineering Sciences, vol. 434, no. 1890, pp. 19-22, 1991.

[18] A. Pasini and V. Pelino, "A unified view of Kolmogorov and Lorenz systems," Physics Letters A, vol. 275, no. 5-6, pp. 435446, 2000.

[19] V. Pelino, F. Maimone, and A. Pasini, "Energy cycle for the Lorenz attractor," Chaos, Solitons \& Fractals, vol. 64, pp. 67-77, 2014.

[20] G. Qi and X. Liang, "Mechanical analysis of Qi four-wing chaotic system," Nonlinear Dynamics, vol. 86, no. 2, pp. 10951106, 2016. 
[21] G. Qi and X. Liang, "Force analysis of Qi chaotic system," International Journal of Bifurcation and Chaos, vol. 26, no. 14, p. 1650237, 2016.

[22] G. Qi and J. Zhang, "Energy cycle and bound of Qi chaotic system," Chaos, Solitons \& Fractals, vol. 99, pp. 7-15, 2017.

[23] G. Qi and X. Liang, "Mechanism and energy cycling of the Qi Four-Wing chaotic system," International Journal of Bifurcation and Chaos, vol. 27, no. 12, p. 1750180, 2017.

[24] G. Qi, "Energy cycle of brushless DC motor chaotic system," Applied Mathematical Modelling, vol. 51, pp. 686-697, 2017.

[25] G. Qi and J. Hu, "Force analysis and energy operation of chaotic system of permanent-magnet synchronous motor," International Journal of Bifurcation and Chaos, vol. 27, no. 14, p. 1750216, 2017.

[26] W. Zhang, "Global and chaotic dynamics for a parametrically excited thin plate," Journal of Sound and Vibration, vol. 239, no. 5, pp. 1013-1036, 2001.

[27] W. Zhang, F. Wang, and M. Yao, "Global bifurcations and chaotic dynamics in nonlinear nonplanar oscillations of a parametrically excited cantilever beam," Nonlinear Dynamics, vol. 40, no. 3, pp. 251-279, 2005.

[28] W. Zhang and Y. Tang, "Global dynamics of the cable under combined parametrical and external excitations," International Journal of Non-Linear Mechanics, vol. 37, no. 3, pp. 505-526, 2002.

[29] M. H. Yao, W. Zhang, and J. W. Zu, "Multi-pulse chaotic dynamics in non-planar motion of parametrically excited viscoelastic moving belt," Journal of Sound and Vibration, vol. 331, no. 11, pp. 2624-2653, 2012.

[30] Y. Zhang, M. Yao, W. Zhang, and B. Wen, "Dynamical modeling and multi-pulse chaotic dynamics of cantilevered pipe conveying pulsating fluid in parametric resonance," Aerospace Science and Technology, vol. 68, pp. 441-453, 2017.

[31] Y. Yang and G. Qi, "Comparing mechanical analysis with generalized-competitive-mode analysis for the plasma chaotic system," Physics Letters A, vol. 383, no. 4, pp. 318-327, 2019.

[32] M. Faramin and M. Ataei, "Chaotic attitude analysis of a satellite via Lyapunov exponents and its robust nonlinear control subject to disturbances and uncertainties," Nonlinear Dynamics, vol. 83, no. 1-2, pp. 361-374, 2016.

[33] J. Wang, X. Liu, and J. Liang, "Bogdanov-Takens bifurcation in an oscillator with positive damping and multiple delays," Nonlinear Dynamics, vol. 87, no. 1, pp. 255-269, 2017.

[34] J. E. Marsden and T. S. Ratiu, Introduction to Mechanics And Symmetry: A Basic Exposition of Classical Mechanical Systems, Springer, 2002. 


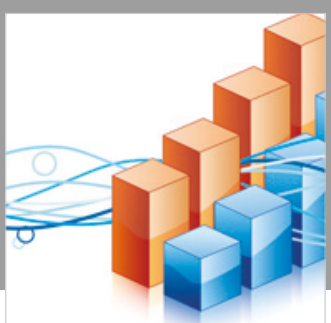

Advances in

Operations Research

\section{-n-m}
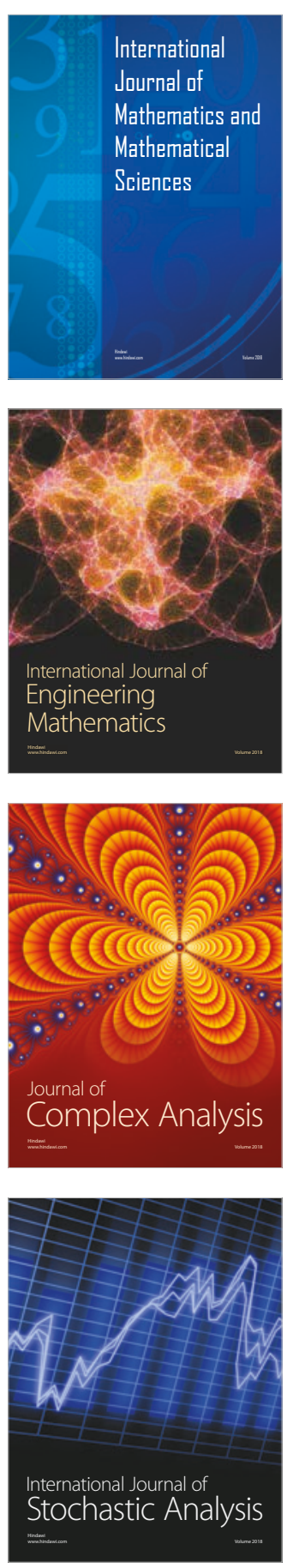
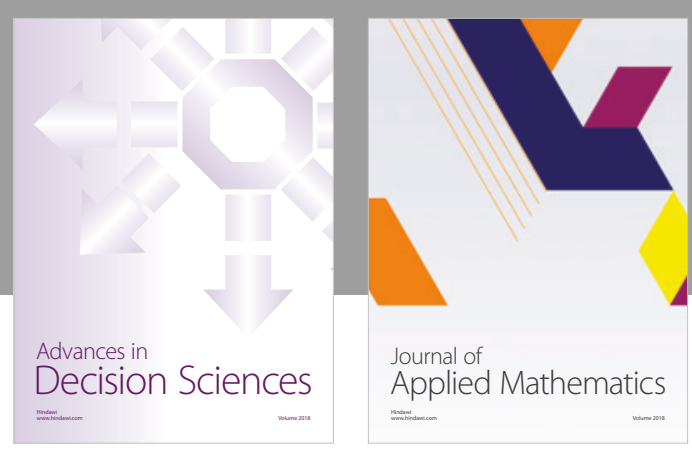

Journal of

Applied Mathematics
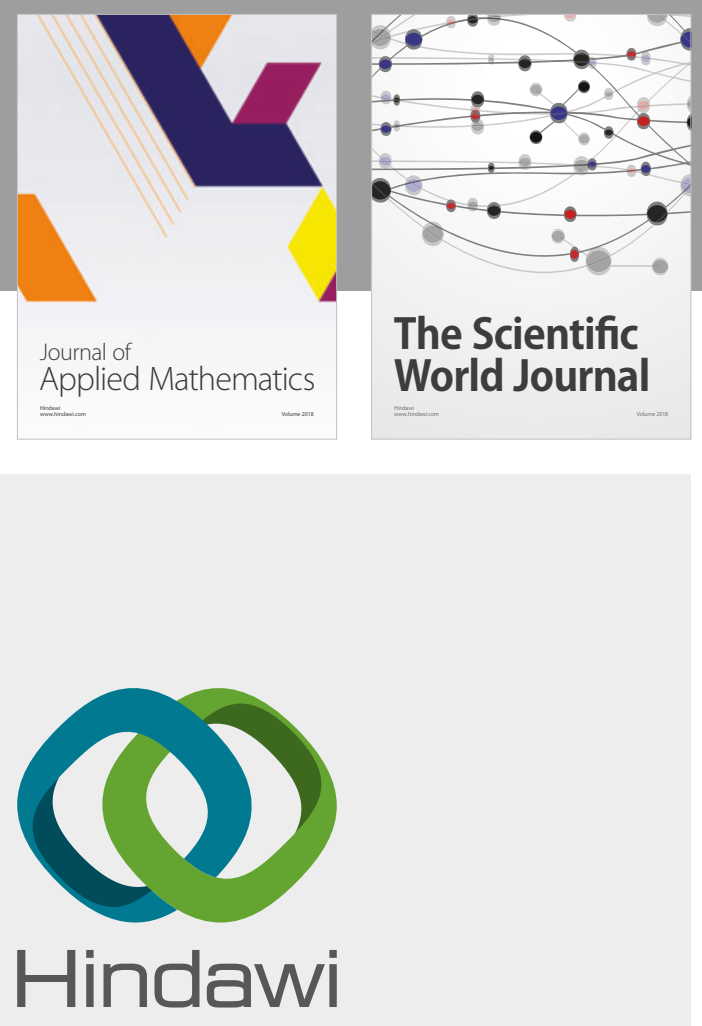

Submit your manuscripts at

www.hindawi.com

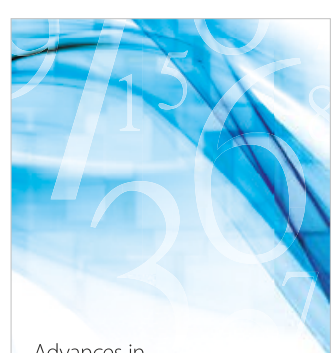

Advances in
Numerical Analysis
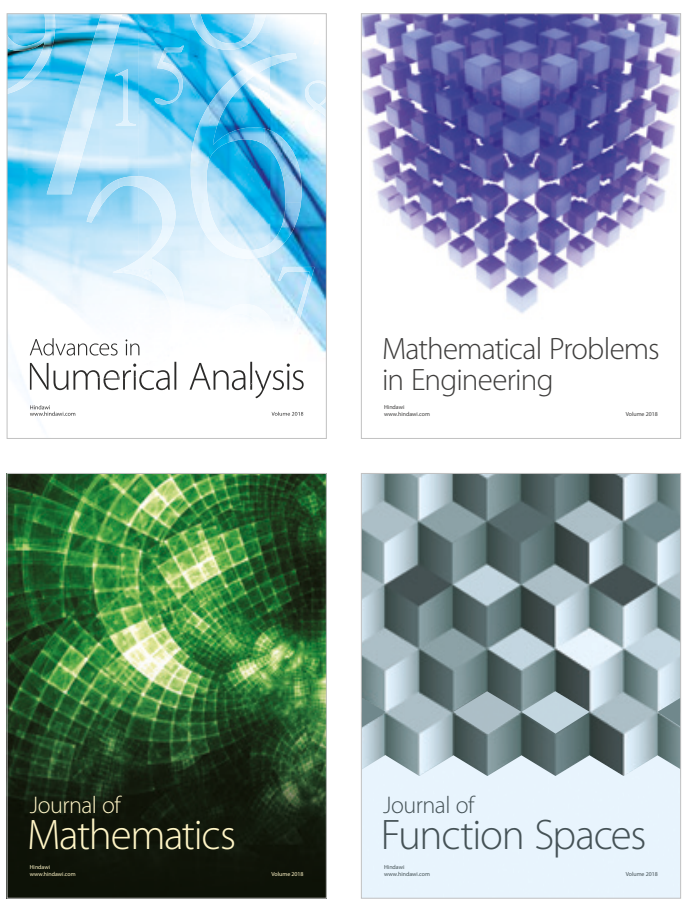

Mathematical Problems in Engineering

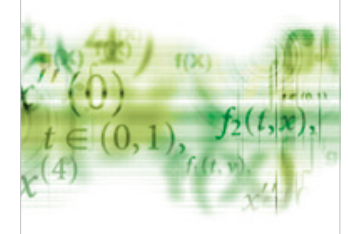

International Journal of

Differential Equations

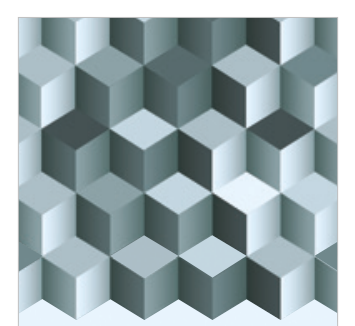

Journal of

Function Spaces
The Scientific

World Journal

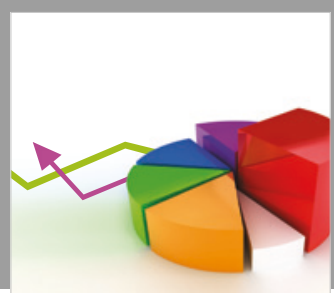

Journal of

Probability and Statistics
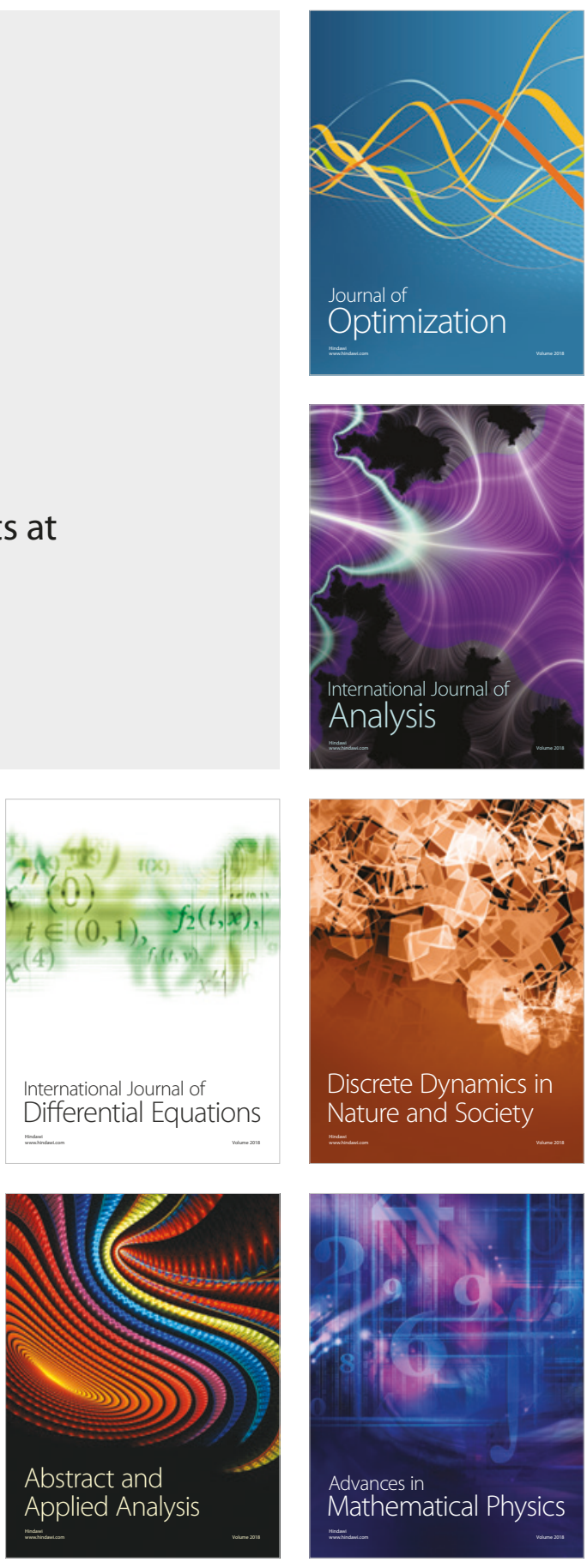